\title{
Binarity of transit host stars ${ }^{\star}$ Implications for planetary parameters
}

\author{
S. Daemgen ${ }^{1}$, F. Hormuth ${ }^{1}$, W. Brandner ${ }^{1}$, C. Bergfors ${ }^{1}$, M. Janson ${ }^{1,2}$, S. Hippler ${ }^{1}$, and T. Henning ${ }^{1}$ \\ 1 Max-Planck-Institut für Astronomie, Königstuhl 17, 69117 Heidelberg, Germany \\ e-mail: daemgen@mpia-hd.mpg.de \\ 2 University of Toronto, Department of Astronomy, St. George Street 50, M5S 3H4 Toronto, ON, Canada
}

Received 19 September 2008 / Accepted 29 January 2009

\section{ABSTRACT}

\begin{abstract}
Context. Straight-forward derivation of planetary parameters can only be achieved in transiting planetary systems. However, planetary attributes such as radius and mass strongly depend on stellar host parameters. Discovering a transit host star to be multiple leads to a necessary revision of the derived stellar and planetary parameters.

Aims. Based on our observations of 14 transiting exoplanet hosts, we derive parameters of the individual components of three transit host stars (WASP-2, TrES-2, and TrES-4) which we detected to be binaries. Two of these have not been known to be multiple before. Parameters of the corresponding exoplanets are revised.

Methods. High-resolution "Lucky Imaging" with AstraLux at the $2.2 \mathrm{~m}$ Calar Alto telescope provided near diffraction limited images in $i^{\prime}$ and $z^{\prime}$ passbands. These results have been combined with existing planetary data in order to recalibrate planetary attributes. Results. Despite the faintness $(\Delta \mathrm{mag} \sim 4)$ of the discovered stellar companions to TrES-2, TrES-4, and WASP-2, light-curve deduced parameters change by up to more than $1 \sigma$. We discuss a possible relation between binary separation and planetary properties, which - if confirmed - could hint at the influence of binarity on the planet formation process.
\end{abstract}

Key words. instrumentation: high angular resolution - stars: binaries: general - planets and satellites: general stars: individual: WASP-2 - stars: individual: TrES-2 - stars: individual: TrES-4

\section{Introduction}

It was not long after the discovery of the first extrasolar planet around another star (Mayor \& Queloz 1995) that the first multiple system was identified among exoplanet hosts. Butler et al. (1997) detected 55 Cnc, $v$ And, and $\tau$ Boo to not only host a planet, but also be bound in binaries. During the following years more and more surveys started to explore exoplanet host multiplicity resulting in the discovery of more than 30 binaries and triples among the exoplanet host sample to date (Mugrauer et al. 2007).

Extensive seeing-limited studies were conducted searching for multiples among exoplanet host stars. Raghavan et al. (2006) used the Sloan Digital Sky Survey (SDSS) to detect 30 exoplanet hosts with one or more stellar companions. Mugrauer et al. (2004) carried out near infrared imaging with the UFTI/UKIRT and SofI/NTT instruments in order to detect wide stellar companions. These early surveys were limited to the seeing limit, confirming theorists' claims that planetary systems in wide binaries are not greatly affected in terms of frequency and parameter statistics (e.g. Desidera \& Barbieri 2007).

Very recent and ongoing high-resolution surveys (Eggenberger et al. 2007; Chauvin et al. 2006; Mugrauer \& Neuhäuser 2005) started to explore smaller separations. Adaptive optics were used to resolve radial velocity (RV) planets into multiple stars where mainly the brighter component

\footnotetext{
* Based on observations collected at the Centro Astronómico Hispano Alemán (CAHA) at Calar Alto, operated jointly by the Max-PlanckInstitut für Astronomie and the Instituto de Astrofísica de Andalucía (CSIC).
}

had been found to host the planet. First results show that binary statistics and parameters of planet hosts are different from those of single stars, implying a modified formation, migration, and/or dynamical evolution process (Desidera \& Barbieri 2007).

Due to its currently more efficient planet detection capability, most ongoing multiplicity surveys concentrate on planet hosts found by RV. However, of today's more than 300 known exoplanets, over 50 were found by photometric surveys due to their transit behavior. Transits (in combination with RV measurements) are the only way to derive the complete set of planetary parameters and therefore deserve special attention. Nonetheless, one has to be careful about parameters deduced, since one cannot rule out the possibility of a fully blended close companion to the exoplanet host star. Although a bright secondary might show up in follow-up spectra as a second set of lines, a faint ( $\Delta$ mag $=$ several magnitudes $)$ long-period companion would not be seen in the spectra as it would be buried in the primary's noise. These companions do not affect spectral parameters such as the RV semi amplitude $K_{*}$ and mass estimates from spectral features, but they demand another evaluation of the transit light curve resulting in a change of planet properties.

In the following we discuss the impact of binarity on transit light curve evaluation and apply this to our findings of three companions to transit hosts, namely WASP-2, TrES-2, and TrES-4.

\section{Sample selection}

Our observations of 14 exoplanet hosts belong to a survey of high-resolution imaging of ultimately all transit exoplanet hosts 
Table 1. Transiting exoplanet hosts observed with AstraLux with no stellar companions found ${ }^{\dagger}$.

\begin{tabular}{lcc}
\hline \hline Name & 2MASS Name & $\mathrm{Vmag}^{a}$ \\
\hline HD 209 458 & $\mathrm{J} 22031077+1853036$ & 7.65 \\
HD 189 733 & $\mathrm{J} 20004370+2242391$ & 7.68 \\
HD 171 56 & $\mathrm{J} 02494447+7145115$ & 8.17 \\
GJ 436 & $\mathrm{J} 11421096+2642251$ & 10.68 \\
WASP-1 & $\mathrm{J} 00204007+3159239$ & 11.8 \\
TrES-1 & $\mathrm{J} 19040985+3637574$ & 11.4 \\
TrES-3 & $\mathrm{J} 17520702+3732461$ & 12.40 \\
HAT-P-1 & $\mathrm{J} 22574684+3840302$ & 10.4 \\
HAT-P-5 & $\mathrm{J} 18173731+3637170$ & 11.95 \\
HAT-P-6 & $\mathrm{J} 23390581+4227575$ & 10.54 \\
XO-2 & $\mathrm{J} 07480647+5013328$ & 11.25 \\
\hline
\end{tabular}

$\dagger$ According to our sensitivity limits (see Sect. 6.1). ${ }^{a} V$ band magnitudes from Simbad.

discovered to date. The survey started in May 2007 observing the first of the $\sim 30$ northern transit planet hosts. Due to its limiting magnitude of $i^{\prime} \approx 15$, all transit hosts in the northern hemisphere are accessible to our observations. The 14 exoplanet hosts that have already been observed are listed in Tables 1 and 2 along with their coordinates and magnitudes.

\section{Observations and data reduction}

\subsection{Direct imaging with AstraLux at Calar Alto}

Observations were obtained with the AstraLux instrument attached to the Cassegrain focus of the $2.2 \mathrm{~m}$ telescope at Calar Alto observatory from May 2007 to November 2007. Reobservations of TrES-4 were conducted in June 2008, since first epoch observations were incomplete. The instrument employs the "Lucky Imaging" technique (Law et al. 2006) which uses several thousand short ( $\sim 10 \mathrm{~ms})$ exposures in order to minimize the effect of atmospheric seeing, coadding only the least distorted images for further reduction. Owing to Lucky Imaging, diffraction limited images can be obtained without the need for adaptive optics (Hormuth et al. 2008, and references therein).

The AstraLux instrument consists of an electron multiplying CCD which produces images with a pixel scale of 46.6 mas/pixel which is resampled to $23.3 \mathrm{mas} /$ pixel through drizzle combination of the best $2.5,5$, and $10 \%$ of the raw images respectively. The full width at half maximum $(F W H M)$ of the final Point Spread Functions (PSF) are on average $0 . ' 1$, similar to with adaptic optics observations at similar sized telescopes.

A more extensive introduction to AstraLux can be found in Hormuth et al. (2008).

\subsection{Photometry and relative astrometry}

Final $i^{\prime}$ and $z^{\prime}$ images were compiled by use of a pipeline which selects the individual images with the highest Strehl ratios and runs the shift-and-add drizzle combination. The final images clearly show a companion to three of our targets (WASP-2, TrES-2, and TrES-4) in separations that place them within the primary's PSF wings. Images of the binaries taken in $z^{\prime}$-band are shown in Fig. 1, coordinates and magnitudes as well as integrated spectral types (SpT) and distances are listed in Table 2. Since no uncertainty estimates are published for the distance of WASP-2, we derive its distance from apparent (Street et al. 2007) and absolute $V$ magnitudes from the SpT $-M_{V}$ relation in
Allen (1999). Uncertainty is estimated from derivation of distance values from upper and lower SpT assuming an uncertainty of one sub class. The value in Table 2 of 147 pc matches earlier determinations and the uncertainty seems to be in a reasonable range.

Separations and position angles of those binaries were determined using the ALLSTAR PSF-fitting task in IRAF. For each target an individual PSF was built in the following way: we determine the size of a rectangle sufficiently sized to contain almost all of the faint companion's flux $\left(\sim 50 \times 50 \mathrm{px} \approx 1^{\prime \prime} .2 \times 11^{\prime \prime} .2\right)$. The content of a box of the same size was then copied from the opposite side of the primary's flux maximum. Rotating this box by $180^{\circ}$ and shifting it to the secondary's position, the flux distribution within the box nicely fits into the surrounding flux distribution replacing the secondary's signature. The resulting PSF consists of the primary's PSF without light from the secondary. In some images - where the PSF decisively deviates from a point symmetric shape - residual "steps" at the edges of the inserted box are visible. However, step sizes never exceed $20 \%$ of the surrounding total flux count and all discontinuities are well separated from the PSF's core. The central region of the PSF, which dominates the photometry results, remains unchanged. The accuracy of this method was tested using PSF stars without contamination by a close companion. Comparing photometry on several objects with and without copying a region according to the above scheme, we did not measure any differences in magnitude and location (nominal display precision $\delta \mathrm{mag}_{\min }=0.001$ ). Hence, the accuracy of the deduced results is only negligibly influenced by this method.

This rather unconventional approach to finding a suitable PSF is preferable over PSF stacking from other observations, as the AstraLux PSF is subject to variations and additional bright sources suitable as reference PSFs have been found in only very few of the science acquisitions. Those have not been used for photometry, in order to consistently evaluate the photometric data.

PSF photometry yields stellar locations with subpixel accuracy. From this, separation and position angle were determined. Astrometric and photometric data are summarized in Table 3.

\section{Stellar properties}

\subsection{Spectral types, magnitudes, and distances}

For WASP-2 and TrES-2, published spectral types of the unresolved binaries are available. However, published spectral types for TrES-4 seem to be inconsistent. We estimate the SpT of TrES-4 from its $J-H$ color using templates by Allen (1999). The resulting SpT of F8 will be used in this paper. In order to determine separate SpTs of the A and B component, integrated SpTs as well as integrated and separate colors were used.

The SpT of the primary component is assumed to be identical to the integrated SpT that was observed without the knowledge about the binarity of the target (see Table 2). This is a reasonable assumption due to the faintness of the companion. As SpTs of all three objects were determined by spectroscopy, a $\Delta$ mag $\approx 4$ secondary does not influence the SpT determination since it is not visible in the spectra. Secondary component SpTs, however, need to be estimated from their color.

A color-SpT diagram ranging from F5 to M5 was composed from template spectra (Pickles 1998) which were convolved with filter curves of the SDSS $z$ and $i$ filters at zero airmass ${ }^{1}$

\footnotetext{
1 http://www . sdss.org/dr3/instruments/imager/index . html
} 
Table 2. Integrated properties of the previously unresolved targets.

\begin{tabular}{lcccccccc}
\hline \hline Name & RA & Dec & $\begin{array}{c}J^{a} \\
{[\mathrm{mag}]}\end{array}$ & $\begin{array}{c}H^{a} \\
{[\mathrm{mag}]}\end{array}$ & $\begin{array}{c}K^{a} \\
{[\mathrm{mag}]}\end{array}$ & \multicolumn{1}{c}{ SpT } & \multicolumn{1}{c}{$\begin{array}{c}d \\
{[\mathrm{pc}]}\end{array}$} & Ref. \\
\hline WASP-2 & 203054.13 & +062546.37 & $10.17 \pm 0.03$ & $9.75 \pm 0.03$ & $9.63 \pm 0.02$ & $\mathrm{~K} 1 \mathrm{~V}$ & $147 \pm 17^{b}$ & 1,2 \\
TrES-2 & 190714.04 & +491859.07 & $10.23 \pm 0.02$ & $9.92 \pm 0.03$ & $9.85 \pm 0.02$ & G0V & $213 \pm 11$ & 3,4 \\
TrES-4 & 175313.06 & +371242.36 & $10.58 \pm 0.02$ & $10.35 \pm 0.02$ & $10.33 \pm 0.02$ & F8 $^{c}$ & $485 \pm 31$ & 4 \\
\hline
\end{tabular}

${ }^{a}$ Magnitudes are from the 2 Micron All Sky Survey (2MASS) catalogue (Skrutskie et al. 2006). ${ }^{b}$ The distance of WASP-2 has been derived indirectly from $V$ band magnitudes (see Sect. 3.2). ${ }^{c}$ Since there are no good values published for TrES-4, we estimate the SpT from the $J-H$ color using templates by Allen (1999). References. (1) Cameron et al. (2007); (2) Allen (1999); (3) Sozzetti et al. (2007); (4) Torres et al. (2008a).
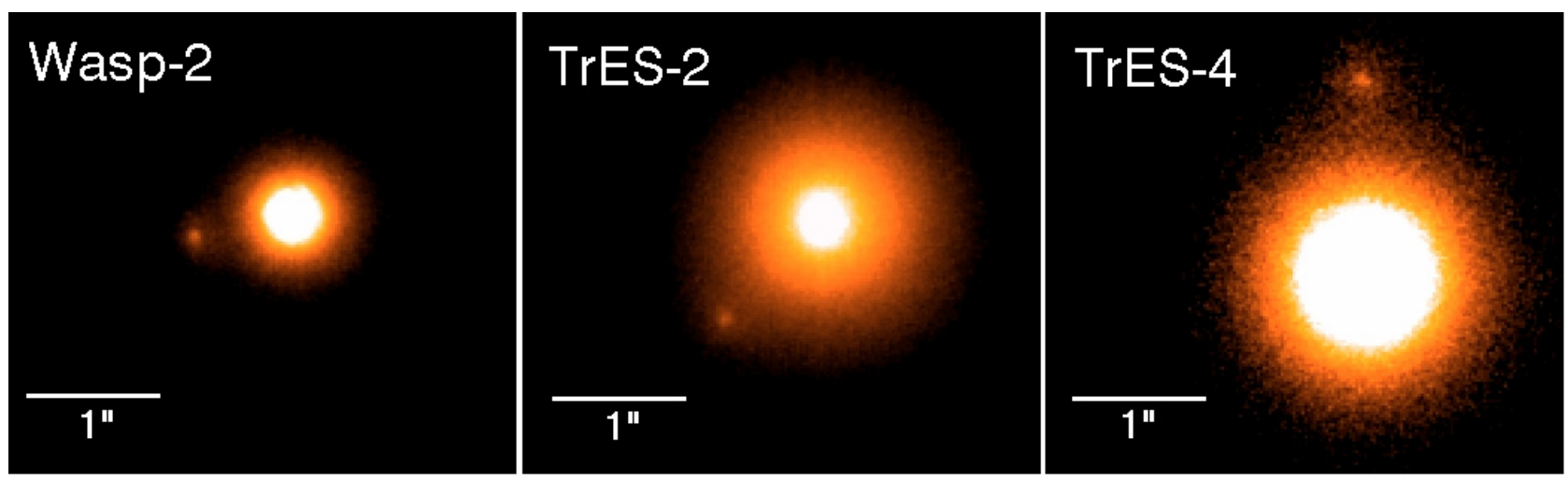

Fig. 1. $z^{\prime}$ filter images of the three exoplanet hosts with AstraLux at the Calar Alto observatory $2.2 \mathrm{~m}$ telescope. Total integration times are $7.68 \mathrm{~s}$ for WASP-2, $15.36 \mathrm{~s}$ for TrES-2, and $11.52 \mathrm{~s}$ for TrES-4. The image scale is linear and cuts are adapted to best depicture the secondary. North is up and East is to the left.

Table 3. Observed binary properties.

\begin{tabular}{lccccc}
\hline \hline Name & $\begin{array}{c}\Delta i^{\prime} \\
{[\mathrm{mag}]}\end{array}$ & $\begin{array}{c}\Delta z^{\prime} \\
{[\mathrm{mag}]}\end{array}$ & $\begin{array}{c}\text { Sep } \\
{\left[{ }^{\prime \prime}\right]}\end{array}$ & $\begin{array}{c}\text { PA } \\
{\left[{ }^{\circ}\right]}\end{array}$ & $\begin{array}{c}\text { Date } \\
(\mathrm{UT})\end{array}$ \\
\hline WASP-2 & $4.095 \pm 0.025$ & $3.626 \pm 0.022$ & $0.757 \pm 0.001$ & $104.7 \pm 0.3$ & Nov. 2007 \\
TrES-2 & $3.661 \pm 0.016$ & $3.429 \pm 0.010$ & $1.089 \pm 0.008$ & $135.5 \pm 0.1$ & May 2007 \\
TrES-4 & $4.560 \pm 0.017$ & $4.232 \pm 0.025$ & $1.555 \pm 0.005$ & $359.8 \pm 0.1$ & Jun. 2008 \\
\hline
\end{tabular}

(Fig. 2). Zeropoints were determined according to the definition of AB magnitudes (Oke 1974). The small correction to convert from $\operatorname{SDSS} i, z$ to $i^{\prime}, z^{\prime}$ magnitudes was applied according to the formula given on the SDSS website. Comparing our synthetic $i^{\prime}-z^{\prime}$ values to those of SDSS main sequence standard stars (from Smith et al. 2002, see Fig. 2), we find that the relation very well fits observed data. We use the $\delta\left(i^{\prime}-z^{\prime}\right)=0.033$ scatter of standard colors about our color-SpT curve as a measure of the $i^{\prime}-z^{\prime}$ uncertainties from our relation. This uncertainty applies to all primary component $\left(i^{\prime}-z^{\prime}\right)_{\mathrm{A}}$ colors which are derived from SpT and our color-SpT relation. The color of the secondary component is calculated through $\left(i^{\prime}-z^{\prime}\right)_{/ \mathrm{C}}=\Delta i^{\prime}-\Delta z^{\prime}-(i-z)_{\mathrm{A}}$ with $\Delta i^{\prime}=i_{/ \mathrm{C}}^{\prime}-i_{\mathrm{A}}^{\prime}$ and $\Delta z^{\prime}=z_{/ \mathrm{C}}^{\prime}-z_{\mathrm{A}}^{\prime}$ from Table 3 , and $\left(i^{\prime}-z^{\prime}\right)_{\mathrm{A}}$ is taken from the A components' values in Table 4.

Reading off spectral types from the $\left(i^{\prime}-z^{\prime}\right)$-SpT relation using our separate $i^{\prime}-z^{\prime}$ colors (see Table 4 ), we receive individual $\mathrm{SpT}$ values with uncertainties of about 1 spectral class for SpTs later than $\sim \mathrm{K} 5$. Individual uncertainties were estimated by determining the SpT from the curve for upper and lower limits of the color measurement respectively. Spectral types earlier than K5 are less reliably determined from the diagram due to the rapidly dropping gradient of $i^{\prime}-z^{\prime}$ color with SpT. However, all secondary spectral types are within or close to the steep region of SpT later than K5. Hence, SpTs are believed to be reasonably well determined by this method.
Since there are no published $i^{\prime}$ and $z^{\prime}$ magnitudes to be found for the unresolved exoplanet hosts we estimate separate $i^{\prime}$ and $z^{\prime}$ magnitudes of A and B components from photometry of reference objects. We use images of the SDSS standards SA 112-223, Ross 711, and BD+71 0031 which we observed during the same night at similar airmass as WASP-2, TrES-2, and TrES-4. This enables us to establish zero points of our $i^{\prime}$ and $z^{\prime}$ exposures by comparing aperture photometry results from the IDL $A T V^{2}$ routine to SDSS standard photometry. These zeropoints are then applied to the science target magnitude measurements.

Apparent magnitude uncertainties are estimated to be $\delta$ mag $\approx 0.10$ for the following reasons. Aperture photometry allows a nominal precision of $\approx 0.03 \mathrm{mag}$ which is derived from the change of measured magnitude when varying the three aperture photometry parameters (aperture radius, annulus radius \& annulus width) within a reasonable range. Converting these numbers into zeropoints and absolute magnitudes increases this uncertainty up to about 0.07 in some cases. Furthermore, exposures are not corrected for airmass, but taken at similar airmasses. We account for that by assuming an additional uncertainty. Hence, total uncertainties of our absolute photometry are conservatively assumed to be $\delta \mathrm{mag} \approx 0.10$. The results are tabulated in Table 4 .

$\overline{{ }^{2} \text { http://www.physics.uci.edu/ barth/atv/ }}$ 


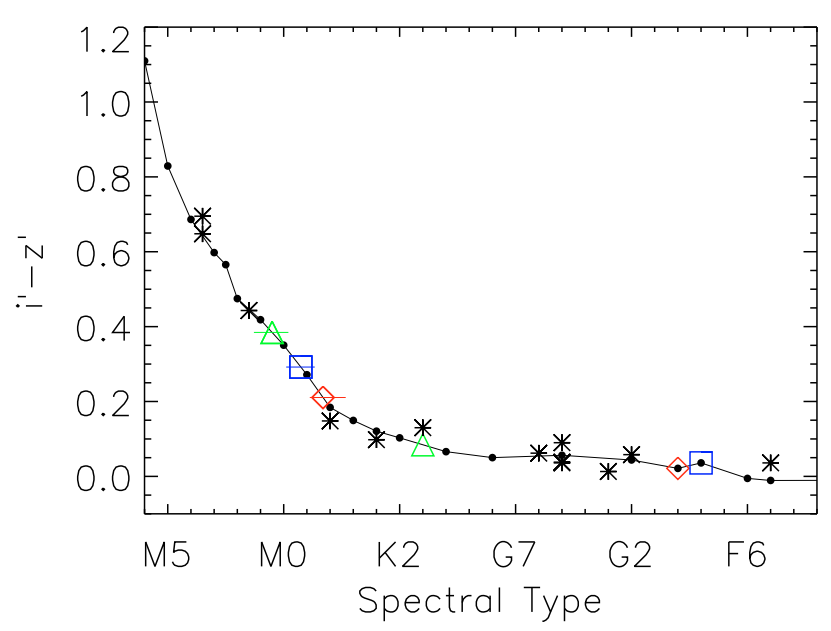

Fig. 2. $i^{\prime}-z^{\prime}$ color vs. spectral type derived from template spectra from Pickles (1998) convolved with the sdss $i^{\prime}$ and $z^{\prime}$ filter curves. Overplotted are the best values for A and B component of three objects: $\Delta$ (WASP-2), $\diamond(\operatorname{TrES}-2)$, and $\square$ (TrES-4). The bars on the three leftmost items denote the spectral uncertainty of the B component of each of the three binaries, respectively. Since we assume an SpT from the literature for the A component no error bar is given. Asterisks (*) denote the positions of SDSS standard main sequence stars from Smith et al. (2002). These are included to verify the accuracy of the relation found as well as to provide an uncertainty estimate of colors derived.

Distances are estimated photometrically from apparent and absolute $M_{i^{\prime}}$ magnitudes. The latter are derived from our SpT estimates and a SpT $-M_{i}$ relation by Kraus \& Hillenbrand (2007) which was converted to $M_{i^{\prime}}$ applying the SDSS conversions. $M_{i^{\prime}}$ values were derived applying cubic spline interpolation in order to reach values that are not explicitly published in their table. Since there are no uncertainties published for the $M_{i^{\prime}}$ curve, we estimate uncertainties for the A components by assuming a SpT uncertainty of one subclass. For the B component we use the spectral uncertainty in Table 4. We derive and compare distances for individual components to decide whether the binaries are physical (see Sect. 6.2). Table 4.

Individual component parameters are summarized in

\subsection{Masses and periods}

None of the stellar secondaries were individually investigated by use of a spectrograph. Hence, deduction of mass from spectral features has not been possible for the separate components. Masses of the individual binary components are estimated according to the following, assuming physical attachment of the binary components. A discussion of physical binarity can be found in Sect. 6.2.

Since the secondary is not observable in the acquired spectra, we assume spectral measurements to correspond to the primary's properties. Hence, characteristics such as mass derived from stellar models (see Torres et al. 2008a, for a consistent discussion of all three objects) need to be attributed to the primary.

Our calculations rely on numbers by Torres et al. (2008a) who collected host mass and radius through comparison of

$$
\left(\frac{a}{R_{*}}\right)_{Y^{2}}=\left(\frac{G}{4 \pi^{2}}\right)^{1 / 3} \frac{P^{2 / 3}}{R_{*}}\left(M_{*}+M_{\mathrm{p}}\right)^{1 / 3}
$$

with input from Yonsei-Yale ( $\left.\mathrm{Y}^{2}\right)$ Models (Yi et al. 2001) and

$$
\left(\frac{a}{R_{*}}\right)_{\text {transit }}=\left\{\frac{\left(1+R_{\mathrm{p}} / R_{*}\right)^{2}-b^{2}\left[1-\sin ^{2}\left(t_{T} \pi / P\right)\right]}{\sin ^{2}\left(t_{T} \pi / P\right)}\right\}^{3 / 2}
$$

entirely fed with light curve parameters. In this paper we derive updated values of $\left(a / R_{*}\right)_{\text {transit }}$ taking the binarity of the transit host star into account (see Sect. 5). However, we expect negligible impact on the deduced primary mass when adapting the models in order to match the new $a / R_{*}$ value: newly derived $a / R_{*}$ are $0.9 \%, 1.0 \%$, and $0.5 \%$ bigger than the previously reported values for WASP-2, TrES-2, and TrES-4 respectively. This translates to a somewhat larger change in mass driven by the $1 / 3$ exponent of the masses in Eq. (1): a $1 \%$ increase of $a / R$ results in a $3 \%$ increase of stellar mass. This is clearly within the uncertainty of the mass estimations of any of the three targets which are of the order of $5-12 \%$. We therefore adopt the mass that was previously attributed to the formerly supposed single star as the primary mass of the newly discovered binary.

In order to estimate a binary period by the use of the Kepler's third law (Kepler et al. 1619), secondary masses are estimated from our SpT according to Allen (1999) (see Table 4). However, the numbers used for the estimation of periods in Table 4 are rough estimates only since masses are calculated for zero age main sequence and do not include the effect of metallicity. Hence, if better values were known, those would have been used for calculations, as is the case for all primary components. Their spectroscopically determined masses are listed in Table 5. To account for random inclinations and eccentricities, projected separations have been multiplied by a factor of 1.26 according to simulations by Fischer \& Marcy (1992). Period estimates and separations are listed in Table 4.

\section{New system parameters of planet and host}

We derive a number of parameters of the planets as well as their host stars. Since earlier calculations did not take the blending with the additional sources into account, system parameters will change with respect to previously published numbers.

Transit light curves offer the greatest opportunity of making fundamental planetary parameters directly accessible. Mass, radius, and other planet properties can be calculated from the four light curve parameters and one additional stellar parameter. The light curve parameters are: Period $P$, transit duration $t_{\mathrm{IV}}-t_{\mathrm{I}}$, ingress/egress duration $t_{\mathrm{II}}-t_{\mathrm{I}}$, and dip depth $\Delta F=$ $\left(F_{\text {no transit }}-F_{\text {transit }}\right) / F_{\text {no transit }}$. From these values a number of planetary and stellar parameters can be derived, these are: $R_{\mathrm{p}} / R_{*}$, $a / R_{*}$, impact parameter $b$, inclination $i$, and stellar density $\rho_{*}$ (cf. Seager \& Mallén-Ornelas 2003), where $R_{\mathrm{p}}$ and $R_{*}$ are the radii of planet and star respectively and $a$ is the semimajor axis of the planet orbit. To resolve the above ratios of system parameters, one additional piece of information is needed. This could be either stellar mass or stellar radius. We choose to use $M_{*}$ since stellar radius is typically less well constrained by stellar models. If we assume stellar mass from the literature, stellar radius can be inferred from $\rho_{*}$ which is known from the light curve parameters.

Additional parameters can be derived with knowledge of the radial velocity amplitude $K_{*}$ and the effective temperature $T_{\text {eff }}$ of the host star. Both numbers can be obtained from spectral measurements which have been acquired for all confirmed transiting planet hosts to date. 
Table 4. Individual binary component parameters.

\begin{tabular}{|c|c|c|c|c|c|c|c|c|c|}
\hline Name $^{a}$ & SpT & $\begin{array}{c}M_{*} \\
{\left[M_{\odot}\right]}\end{array}$ & $\begin{array}{c}\left(i^{\prime}-z^{\prime}\right) \\
{[\mathrm{mag}]}\end{array}$ & $\begin{array}{c}i^{\prime b} \\
{[\mathrm{mag}]}\end{array}$ & $\begin{array}{c}z^{\prime b} \\
{[\mathrm{mag}]}\end{array}$ & $\begin{array}{c}M_{i^{\prime}} \\
{[\mathrm{mag}]}\end{array}$ & $\begin{array}{l}d_{\text {phot }} \\
{[\mathrm{pc}]}\end{array}$ & $\begin{array}{l}\mathrm{sep}^{c} \\
{[\mathrm{AU}]}\end{array}$ & $\begin{array}{c}P^{c, d} \\
{\left[10^{3} \mathrm{yr}\right]}\end{array}$ \\
\hline WASP-2A & K1 & 0.77 & $0.084 \pm 0.033$ & 11.27 & 11.19 & $5.74 \pm 0.19$ & $127 \pm 13$ & $111 \pm 13$ & 1.4 \\
\hline WASP-2/C & M0 . . M1.5 & 0.48 & $0.385 \pm 0.047$ & 15.38 & 14.81 & $7.95 \pm 0.29$ & $310 \pm 40$ & & \\
\hline TrES-2A & G0 & 1.05 & $0.021 \pm 0.033$ & 11.07 & 11.04 & $4.45 \pm 0.11$ & $211 \pm 14$ & $232 \pm 12$ & 3.9 \\
\hline TrES-2/C & K4.5 . . K6 & 0.67 & $0.211 \pm 0.038$ & 14.73 & 14.47 & $6.73 \pm 0.20$ & $400 \pm 40$ & & \\
\hline TrES-4A & F8 & 1.18 & $0.036 \pm 0.033$ & 9.33 & 9.29 & $4.27 \pm 0.18$ & $103 \pm 10$ & $755 \pm 49$ & 21 \\
\hline TrES-4/C & K5.5 ... M0 & 0.59 & $0.292 \pm 0.045$ & 13.85 & 13.57 & $7.35 \pm 0.54$ & $200 \pm 50$ & & \\
\hline
\end{tabular}

${ }^{a}$ The stellar secondary is referred to as "/C" in order to avoid confusion with the notation of the planetary companion, which is referred to as an appendix "b" to the host star's name in many publications. ${ }^{b}$ Uncertainties of apparent magnitudes are estimated to be $\delta$ mag $=0.10$ (see Sect. 4.1 ). ${ }^{c}$ Separation and period estimates use distances from Table 2. ${ }^{d}$ Periods are order-of-magnitude estimations and hence do not include an uncertainty estimation.

Table 5. Literature values used to calculate the new parameters.

\begin{tabular}{cccc}
\hline \hline Parameter & WASP- 2 & TrES- & TrES-4 \\
\hline$\Delta F^{a} \ldots \ldots \ldots \ldots \ldots \ldots \ldots$ & $0.01713 \pm 0.00020$ & $0.01570 \pm 0.00013$ & $9.806941 \pm 0.000087$ \\
$P($ days $) \ldots \ldots \ldots \ldots \ldots$ & $2.152226 \pm 0.000004$ & $2.47063 \pm 0.00001$ & $3.553945 \pm 0.000075$ \\
$t_{\mathrm{IV}}-t_{\mathrm{I}}(\mathrm{h}) \ldots \ldots \ldots \ldots \ldots$ & $1.799 \pm 0.035$ & $1.840 \pm 0.020$ & $3.638 \pm 0.0824^{b}$ \\
$t_{\mathrm{II}}-t_{\mathrm{I}}(\mathrm{h}) \ldots \ldots \ldots \ldots \ldots$ & $0.41 \pm 0.04$ & $0.683 \pm 0.045$ & $0.701 \pm 0.026^{b}$ \\
$K\left(\mathrm{~m} \mathrm{~s}^{-} 1\right) \ldots \ldots \ldots \ldots \ldots$ & $155 \pm 7$ & $181.3 \pm 2.6$ & $97.4 \pm 7.2$ \\
$T_{\text {eff }}(\mathrm{K}) \ldots \ldots \ldots \ldots \ldots$ & $5200 \pm 200$ & $5850 \pm 50$ & $6200 \pm 75$ \\
$M_{*}\left(M_{\odot}\right) \ldots \ldots \ldots \ldots \ldots$ & $0.89 \pm 0.12$ & $0.983 \pm 0.061$ & $1.394 \pm 0.058$ \\
Ref. $\ldots \ldots \ldots \ldots \ldots \ldots$ & 1,2 & 3,4 & 5,6 \\
\hline
\end{tabular}

${ }^{a} \Delta F=\left(F_{\text {notransit }}-F_{\text {transit }}\right) / F_{\text {notransit }}=\left(R_{\mathrm{p}} / R_{*}\right)^{2} ;{ }^{b}$ transit duration numbers for TrES-4 are inferred from values published by Mandushev et al. (2007) using inverted formulas from Seager \& Mallén-Ornelas (2003). References. (1) Cameron et al. (2007); (2) Charbonneau et al. (2007); (3) O’Donovan et al. (2006); (4) Holman et al. (2007); (5) Mandushev et al. (2007); (6) Torres et al. (2008a).

\subsection{Impact of host binarity on stellar and planetary parameters}

The evaluation of the transit light curve generally assumes host singularity. Subtracting the constant flux offset of a blended binary component, $\Delta F$ will assume a greater value than $\Delta F_{\text {old }}$ which has been calculated disregarding an existing blend. The new $\Delta F$ is derived through

$\Delta F=\left(1+10^{-\frac{\Delta z^{\prime}}{2.5}}\right) \cdot \Delta F_{\text {old }}$

where $\Delta z^{\prime}$ is the magnitude difference between the primary and the secondary stellar component of the exoplanet host in SDSS $z^{\prime}$ filter (see Table 3).

The formula assumes the brighter component to be the variable component. Provided that the fainter companion was the variable part, an eclipsing binary would be - at first sight - a valid solution. This, however, has been ruled out by line-bisector analysis for all three objects (Cameron et al. 2007; O'Donovan et al. 2006; Mandushev et al. 2007). Additionally, in the case of WASP-2 the necessary $\sim 1.5$ mag eclipse of the secondary in $H$ band in order to explain the light curve could not be observed (Cameron et al. 2007).

For further evaluation, we use formulae (6) through (9) in Seager \& Mallén-Ornelas (2003) which analytically connect $R_{\mathrm{p}} / R_{*}, b, a / R_{*}$, and $\rho_{*}$ to light curve parameters. Further system parameters (planetary surface gravity $\log g_{\mathrm{p}}$, Safronov number $\Theta$, and equilibrium temperature $T_{\text {eq }}$ ) are derived according to the formulae given in Torres et al. (2008a).

Reported uncertainties are copied from published values in the observers' papers. Since the updated numbers in this paper differ by less than $2 \%$ from the old ones, the uncertainties are expected to change in a similar manner on a scale of not more than a few percent. We therefore keep the previous uncertainties since our computational approach cannot reproduce uncertainties with all observational constraints that have been included in the original papers.

Input values used are assembled in Table 5. The resulting set of new parameters is listed in Table 6.

\subsection{Notes on the individual objects}

\subsubsection{WASP-2}

WASP-2b is the second bona fide transiting planet detected by the SuperWASP survey (Pollacco et al. 2006) and followed up with the SOPHIE spectrograph (Bouchy \& The Sophie Team 2006) at the Observatoire de Haute Provence. The discovery paper (Cameron et al. 2007) presents a $R_{\mathrm{p}}=(0.65-1.26) R_{\mathrm{Jup}}$, $M_{\mathrm{p}}=(0.81-0.95) M_{\text {Jup }}$ planet on a 2.15 day orbit around WASP-2.

Cameron et al. (2007) report a stellar companion to WASP-2 0.7 to the east which we confirm with our finding of a $\Delta i^{\prime}=$ $4.095 \pm 0.025, \Delta z^{\prime}=3.626 \pm 0.022$ companion at a consistent position. However, removing the secondary light from the light curve evaluation requires us to augment the former value of $R_{\mathrm{p}} / R_{*}$ by $1.76 \%$, which is greater than the absolute uncertainty of $0.0015 \mathrm{mag}$. This results in a $0.9 \%$ increase of absolute planet radius $R_{\mathrm{p}}$.

\subsubsection{TrES-2}

This transit host with the alternative identifier GSC 03549-02811 was discovered in 2006 by the Trans-Atlantic Exoplanet Survey (TrES; O'Donovan et al. 2006). Due to the smallness of reported uncertainties in the discovery paper and a later refinement of parameters by Holman et al. (2007), the relative change of 
Table 6. The new system parameters.

\begin{tabular}{|c|c|c|c|}
\hline Parameter & $\overline{\text { WASP-2 }}$ & $\overline{\text { TrES-2 }}$ & $\overline{\overline{T r E S}-4}$ \\
\hline$R_{\mathrm{p}} / R_{*} \ldots \ldots \ldots \ldots \ldots \ldots$ & $0.1332 \pm 0.0015$ & $0.1279 \pm 0.0010$ & $0.1000 \pm 0.0009$ \\
\hline$a / R_{*} \ldots \ldots$ & $8.01 \pm 0.32$ & $7.65 \pm 0.12$ & $6.05 \pm 0.13$ \\
\hline b.. & $0.725 \pm 0.026$ & $0.851 \pm 0.006$ & $0.752 \pm 0.015$ \\
\hline$i(\operatorname{deg})$ & $84.80 \pm 0.39$ & $83.62 \pm 0.14$ & $82.86 \pm 0.33$ \\
\hline$\rho_{*}\left(\mathrm{~g} / \mathrm{cm}^{3}\right)$. & $2.095 \pm 0.205$ & $1.390 \pm 0.060$ & $0.333 \pm 0.022$ \\
\hline $\log g_{\mathrm{p}} \ldots \ldots$ & $3.279 \pm 0.036$ & $3.284 \pm 0.016$ & $2.867 \pm 0.038$ \\
\hline$\ldots \ldots \ldots \ldots \ldots$ & $0.843 \pm 0.063$ & $0.999 \pm 0.033$ & $1.809 \pm 0.064$ \\
\hline$R_{\mathrm{p}} / R_{\text {Jup }}$. & $1.117 \pm 0.082$ & $1.272 \pm 0.041$ & $1.799 \pm 0.063$ \\
\hline$M_{\mathrm{p}} / M_{\text {Jup }}$ & $0.914 \pm 0.092$ & $1.199 \pm 0.052$ & $0.919 \pm 0.073$ \\
\hline$\rho_{\mathrm{p}}\left(\mathrm{g} / \mathrm{cm}^{3}\right) \ldots \ldots$ & $0.87 \pm 0.24$ & $0.77 \pm 0.09$ & $0.209 \pm 0.029$ \\
\hline & $0.0590 \pm 0.0044$ & $0.0697 \pm 0.0022$ & $0.0381 \pm 0.0030$ \\
\hline$T_{\text {eq }}(\mathrm{K})$ & $1300 \pm 54$ & $1495 \pm 17$ & $1782 \pm 29$ \\
\hline$\ldots \ldots \ldots \ldots \ldots$ & $0.03138 \pm 0.00142$ & $0.03556 \pm 0.00075$ & $0.05091 \pm 0.00071$ \\
\hline
\end{tabular}

parameters in terms of significance is the biggest for the TrES-2 system. We calculate a $2.1 \%$ increase of $R_{\mathrm{p}} / R_{*}$ which is more than twice the uncertainty of its absolute value. Other parameters change up to $0.7 \sigma$ with respect to previously derived values.

\subsubsection{TrES-4}

The transiting planet TrES-4b is worth mentioning for several reasons. Having been discovered by Mandushev et al. (2007) to orbit the late F star GSC 02620-00648 it was classified as the least dense of all transiting planets known at the time of writing, due to its large radius of $R_{\mathrm{p}}=1.80 \pm 0.06 R_{\text {Jup }}$ (this paper). Similarly, a low value for the parent star's density is calculated. Furthermore, TrES-4 is the only object in our 3-object sample that belongs to the "Class II" objects of low Safronov numbers $\Theta$ at high equilibrium temperatures $T_{\text {eq }}$ (Hansen \& Barman 2007). Implications to draw from $\Theta$ are discussed in Sect. 6.4.

\section{Discussion}

\subsection{Sensitivity limit to the detection of stellar companions}

Table 1 lists all observed transiting planet hosts observed in our 2007 run that do not show signs of stellar companions. We can exclude companions according to our sensitivity limits discussed below.

The dim end of detectable point-like sources is determined by the resolution and background noise of our AstraLux observations. With an average $F W H M$ of the PSF of $\sim 00^{\prime} 1$, the detection of sources several arcseconds away from a bright source is limited by the detection limit of $\Delta i^{\prime} \lesssim 8$ of our AstraLux observations.

In the proximity (comparable to the $F W H M$ of $\sim 0$. $^{\prime} 1$ ) of a star, however, the detection limit is dominated by the photon noise of the primary source. Hence, the minimum detectable magnitude is greater the tighter the binary. Since deriving binary statistics is not the primary goal of this paper, we do not develop a thorough sensitivity examination for very tight binaries and discontinue further evaluation here.

We would like to note that close-in bright companions would have shown up in previously observed spectra. From this constraint a limiting magnitude could be formulated. However, this is dependent on the spectrograph used and hence cannot be discussed in detail here. For binaries with separations greater than or comparable to the $F W H M$, this limit is less strong than our detection limit which pushes the mass down to or even below the deuterium burning limit. The non-detection of binaries from spectroscopy, however, excludes binaries with projected separations closer than possible to resolve with imaging techniques.

Because of a limited field of view of $\sim 12^{\prime \prime} \times 12^{\prime \prime}$ and in order to be complementary to previous observations we chose to restrict binary detections to separations $\lesssim 2^{\prime \prime}$. Wide binaries separated by several arcseconds would have been resolved even with lower than near diffraction-limited resolution; seeing-limited observations have been made for all confirmed transiting exoplanet hosts. In the course of those observations it has been found that two objects, HD 189733 and HAT-P-1, have wide companions (Bakos et al. 2006, 2007). Since separations of both objects are more than $10^{\prime \prime}$ they could not have been detected with our instrumental setup nor would they have passed our separation selection criterion. Nevertheless we include those two findings in our analysis in Sect. 6.4, since they helpfully enlarge the sample of transit host binaries in order to draw more reliable statistical conclusions.

\subsection{Are the companions physically bound?}

Kraus \& Hillenbrand (2007) present absolute magnitudes in the SDSS ugriz filter system allowing us to derive $M_{u^{\prime}} M_{g^{\prime}} M_{r^{\prime}} M_{i^{\prime}} M_{z^{\prime}}$ absolute magnitudes (by applying the conversion formulae) and hence distances for each of the individual target components. Individual distances are used to test whether the components reside at the same distance to Earth and are therefore physically related. Results (Table 4) seem to show a discrepancy between each component pair, for example WASP-2A is calculated at a distance of $127 \pm 13 \mathrm{pc}$ whereas WASP-2/C is suggested to lie at $310 \pm 40 \mathrm{pc}$ distance. However, distance uncertainties are probably underestimated due to missing absolute magnitude uncertainties from Kraus \& Hillenbrand (2007) who derive their $M_{i^{\prime}}$ values from a multitude of color-SpT and colorcolor relations.

Since all three objects show a secondary distance of about two times the primary distance, systematic errors might play a role. For example, the absolute photometry assumes the objects to reside on the main sequence. TrES-4, with the greatest discrepancy between $V$ band photometric distance (Table 2) and distance derived from $i^{\prime}$ magnitudes (Table 4), was suggested by Mandushev et al. (2007) to have slightly evolved from the main sequence towards the subgiant branch, which would explain the high $i^{\prime}$ magnitude leading to the small photometric distance. Hence, although photometric distances suggest non-related binary components, we cannot conclude on physical binarity. 
A statistical approach can be carried out by estimating the density of background sources and deriving the expected number of targets accompanied by a background giant within $2^{\prime \prime}$. Since the sky coverage of the SDSS catalog is not sufficient to cover all targets in the sample, we used the 2MASS catalog in order to find all sources within $30^{\prime}$ of each target. The density of giants $\rho\left(m_{K}\right)$ detectable with AstraLux can be estimated with the following cuts. Colors of $J-K \geq 0.5$ are selected to include mostly giants while the limiting magnitude of AstraLux translates into roughly $m_{K} \approx 14$. Applying these cuts we find the probability of detecting a background source brighter than $m_{K}$ within a separation of $\Theta$ according to (see Brandner et al. 2000)

$P\left(\Theta, m_{K}\right)=1-\mathrm{e}^{-\pi \rho\left(m_{K}\right) \Theta^{2}}$.

The average probability of finding one or more giants close to a target is $P=0.16 \%$ according to Eq. (4). Hence, the expected number of sources with non-related background companions is $E=0.022$, which strongly suggests that the three companion sources in this sample are physically attached.

Cameron et al. (2007) report a stellar companion to WASP-2 that is likely to be identical to our observed companion. Their high-resolution observations in September 2006 indicate a $\Delta H=$ 2.7 companion located 0.7 to the East which agrees with our observed PA of $(104.7 \pm 0.3)^{\circ}$ and separation of $0.757 \pm 00^{\prime} 001$ in November 2007. WASP-2, with its proper motion of $53.2 \mathrm{mas} / \mathrm{yr}$ (Zacharias et al. 2004) and a time baseline of $\sim 1 \mathrm{yr}$, is expected to show a significant change in separation if the components are unrelated. However, since there are no uncertainties published we cannot definitively state that WASP-2 and its faint companion share a proper motion.

Despite the above considerations, a reliable confirmation or rejection of physical binarity can only be achieved by second epoch data with a sufficiently long time baseline.

We would like to point out that physical binarity is irrelevant for derivations in Sects. 5, 6.1, and 6.3 which discuss the impact of blending on planetary and stellar parameters. Calculations depend on the contamination by sources with a small angular separation from the target, regardless of their actual distance.

\subsection{The new parameters}

In the following we will refer to values that were derived without knowledge about the host star's binarity as "old", whereas our newly derived values from which the secondary contribution has been removed are referred to as "new".

The change in $\Delta F$ propagates through all numbers derived from the light curve and causes changes of up to more than $3 \%$. Quantifying the change in terms of significance, we find deviations of the new values compared to the old ones of up to more than $2 \sigma$ for the value of $R_{\mathrm{p}} / R_{*}$. Other parameters (listed in Table 6) needed to be revalued by up to $1 \sigma$.

The non-random nature of this effect makes deviances significant although they are $2 \sigma$ and less. For example, in the presence of a companion, $R_{\mathrm{p}} / R_{*}$ can only be greater than assumed before. The size of augmentation is determined by the brightness of the companion. A brighter companion causes a larger change of the parameter. Hence the new parameters imply a shift of the mean value.

The relative uncertainties of the TrES-2 parameters are smaller than for the other two targets. This makes its characteristics subject to the most significant revisions.

\subsection{Planets in the binary environment}

In an attempt to identify correlations between various planetary parameters and host star properties, Hansen \& Barman (2007) also compute the equilibrium temperature and Safronov number, which is defined as $\Theta=\left(a / R_{\mathrm{p}}\right)\left(M_{\mathrm{p}} / M_{*}\right)$, for a sample of 20 transiting exoplanets. They identify two classes of exoplanets based on their Safronov numbers. Class I planets have $\Theta=0.07 \pm 0.01$ and Class II planets $\Theta=0.04 \pm 0.01$. This distinction into two classes of exoplanets is confirmed by Torres et al. (2008b), who increased the sample to 23 sources, and also took stellar evolution into account when computing the physical properties. Hansen \& Barman (2007) speculate that the two classes might be the outcome of different migration mechanisms. Another possibility discussed is that a decrease in the average density due to mass loss by selective evaporation of of helium could explain the presence of Class II planets with the abnomally large radii.

From literature studies ${ }^{3}$, we could find 35 transiting planets for which Safronov numbers could be calculated. In Fig. 3a) we show the 31 transit planets with Safronov numbers smaller than 0.1. The distinction between the Class I and Class II planets at $\Theta \approx 0.05$ is still present. Interestringly, three of the 31 exoplanets are located above the former upper limit for Class I at $\Theta \leq 0.08$. These three might either form yet another group ("Class III"?) or could be seen as an extension of Class I. In Fig. 3b) we show the full range of Safronov numbers for the 35 transit planets, which range up to $\Theta \approx 1.2$. The planets with the highest Safronov numbers are in general the ones with the highest planetary mass relative to the stellar mass.

Of the stellar binaries in our sample, the exoplanets around WASP-2 and TrES-2 belong to Class I, whereas the exoplanet TrES-4b belongs to Class II. As the update from our calculations is small, the membership of the three objects as to Class I and II did not change. From the literature we identify two more binaries, HD 189733 (Class I) and HAT-P-1 (Class II), which both have larger angular separations than the detection cut-off assumed for the AstraLux sample.

Interestingly, for these five objects, there might also be a correlation between the binary separation and the planet class. The three binaries with projected separations between 110 and 230 AU host Class I exoplanets, whereas the wider binaries with their projected separations of $\geq 750$ AU host Class II exoplanets. The presence of a binary companion could very well influence the planet formation process as well as the predominant migration mechanism.

Clearly our sample of five potential stellar companions of transit planet host stars is too small to draw any firm conclusions. Yet it would be worthwhile to explore if this correlation still holds once the binary sample has been expanded.

\section{Summary}

High-resolution imaging of 14 exoplanet hosts stars revealed stellar companions to three of the targets (WASP-2, TrES-2, and TrES-4), the latter two of which have not been known to be multiple before.

Observations are part of an ongoing imaging survey aiming at characterizing all transit host stars with the high-resolution imager AstraLux. It employs the Lucky Imaging technique which allows spatial resolutions comparable to adaptive optics observations with much smaller overhead and therefore high observing efficiency.

\footnotetext{
${ }^{3}$ References in http://www . exoplanet.eu/
} 

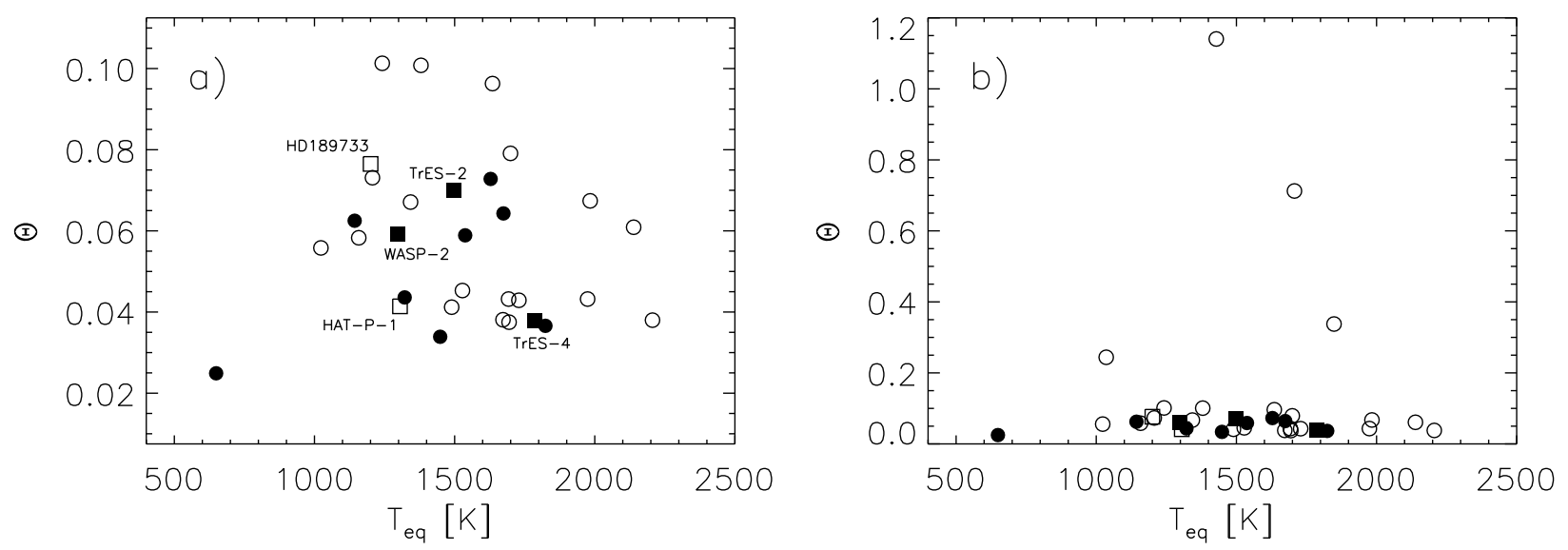

Fig. 3. a) Safronov number $\Theta$ vs. equilibrium temperature $T_{\text {eq }}$ for 31 transiting exoplanet systems. Circles show the position of apparently single systems, whereas squares denote known binaries. Objects marked with filled symbols have been observed in the course of the survey presented in this paper. Names in the plot identify the binary transit hosts. b) Same as a) but with a different scale for $\Theta$ showing four additional objects with large Safronov numbers.

We present an analysis of the photometry and astrometry obtained from our observations. Together with transit light curves and spectroscopic parameters, planetary and stellar parameters were derived and compared with earlier derivations.

We find the companions to be classified as $\mathrm{K} 7$ to M0.5, with a projected separation of 100 to $750 \mathrm{AU}$ to their F8 to K1 candidate parent stars assuming physically bound companions. While physical binarity has yet to be confirmed through second epoch observations, it is not crucial to most of the conclusions drawn.

Analyzing stellar and planetary parameters taking the newly discovered binarity into account we find values to differ from previously derived parameters assuming a single host star: all parameters (e.g. $R_{\mathrm{p}} / R_{*}, a / R_{*}, b, \rho_{*}, \ldots$ ) have undergone revision and updated values have been shown to differ by up to more than $2 \sigma$, where $\sigma$ is the corresponding uncertainty of each parameter.

A correlation between the Safronov number $\Theta$ and binary separation has been suggested. Wide binaries seem to fall into Class II $(\Theta \approx 0.04)$ whereas Class I $(\Theta \approx 0.07)$ seems to host smaller separation binaries in a $\Theta-T_{\text {eq }}$ plot. The very small number of known multiple transit host binaries, however, needs to be increased through future observing in order to confirm or reject this proposal.

Acknowledgements. The authors would like to thank the referee, Dr. Hans Deeg, for many helpful suggestions and comments which lead to a highly improved paper. This publication makes use of data products from the Two Micron All Sky Survey, which is a joint project of the University of Massachusetts and the Infrared Processing and Analysis Center/California Institute of Technology, funded by the National Aeronautics and Space Administration and the National Science Foundation. This research has made use of the SIMBAD database, operated at CDS, Strasbourg, France.

\section{References}

Allen's Astrophysical Quantities 1999, 4th edn, ed. A. N. Cox (Springer Verlag) Bakos, G. Á., Pál, A., Latham, D. W., Noyes, R. W., \& Stefanik, R. P. 2006, ApJ, 641, L57
Bakos, G. Á., Noyes, R. W., Kova'cs, G., et al. 2007, ApJ, 656, 552 Bouchy, F., \& The Sophie Team 2006, Tenth Anniversary of 51 Peg-b: Status of and prospects for hot Jupiter studies, 319

Brandner, W., Zinnecker, H., Alcalá, J. M., et al. 2000, AJ, 120, 950

Butler, R. P., Marcy, G. W., Williams, E., Hauser, H., \& Shirts, P. 1997, ApJ, 474, L115

Cameron, A. C., Bouchy, F., He'brard, G., et al. 2007, MNRAS, 375, 951 Charbonneau, D., Winn, J. N., Everett, M. E., et al. 2007, ApJ, 658, 1322

Chauvin, G., Lagrange, A.-M., Udry, S., et al. 2006, A\&A, 456, 1165 Desidera, S., \& Barbieri, M. 2007, A\&A, 462, 345

Eggenberger, A., Udry, S., Chauvin, G., et al. 2007, A\&A, 474, 273

Fischer, D. A., \& Marcy, G. W. 1992, ApJ, 396, 178

Hansen, B. M. S., \& Barman, T. 2007, ApJ, 671, 861

Holman, M. J., Winn, J. N., Latham, D. W., et al. 2007, ApJ, 664, 1185

Hormuth, F., Hippler, S., Brandner, W., Wagner, K., \& Henning, T. 2008, Proc. SPIE, 7014, 701448

Kepler, J., Ptolemaeus, C., \& Fludd, R. 1619, Lincii Austriae, sumptibus G. Tampachii, excudebat I. Plancvs

Kraus, A. L., \& Hillenbrand, L. A. 2007, AJ, 134, 2340

Law, N. M., Mackay, C. D., \& Baldwin, J. E. 2006, A\&A, 446, 739

Mandushev, G., O’Donovan, F. T., Charbonneau, D., et al. 2007, ApJ, 667, 195

Mayor, M., \& Queloz, D. 1995, Nature, 378, 355 L195

Mugrauer, M., \& Neuhäuser, R. 2005, MNRAS, 361, L15

Mugrauer, M., Neuhäuser, R., Mazeh, T., Fernández, M., \& Guenther, E. 2004, The Search for Other Worlds, 713, 31

Mugrauer, M., Seifahrt, A., \& Neuhäuser, R. 2007, MNRAS, 378, 1328

O'Donovan, F. T., Charbonneau, D., Mandushev, G., et al. 2006, ApJ, 651, L61

Oke, J. B. 1974, ApJS, 27, 21

Pickles, A. J. 1998, PASP, 110, 863

Pollacco, D. L., Skillen, I., Cameron, A. C., et al. 2006, PASP, 118, 1407

Raghavan, D., Henry, T. J., Mason, B. D., et al. 2006, ApJ, 646, 523

Seager, S., \& Mallén-Ornelas, G. 2003, ApJ, 585, 1038

Skrutskie, M. F., Cutri, R. M., Stiening, R., et al. 2006, AJ, 131, 1163

Smith, J. A., Tucker, D. L., Kent, S., et al. 2002, AJ, 123, 2121

Sozzetti, A., Torres, G., Charbonneau, D., et al. 2007, ApJ, 664, 1190

Street, R. A., Christian, D. J., Clarkson, W. I., et al. 2007, MNRAS, 379, 816

Torres, G., Winn, J. N., \& Holman, M. J. 2008a, ApJ, 677, 1324

Torres, G., Winn, J. N., \& Holman, M. J. 2008b [arXiv: 0806. 4353]

Yi, S., Demarque, P., Kim, Y.-C., et al. 2001, ApJS, 136, 417

Zacharias, N., Urban, S. E., Zacharias, M. I., et al. 2004, AJ, 127, 3043 